Supporting Information of

\title{
The Role of Range-Separated Correlation in Long-Range Corrected Hybrid Functionals
}

\author{
Wenna $\mathrm{Ai}^{1}$, Wei-Hai Fang ${ }^{1}$ and Neil Qiang $\mathrm{Su}^{*, 1}$ \\ ${ }^{1}$ Department of Chemistry, Key Laboratory of Advanced Energy Materials Chemistry (Ministry of \\ Education) and Renewable Energy Conversion and Storage Center (RECAST), Nankai University, \\ Tianjin 300071, China
}

*E-mail: nqsu@nankai.edu.cn

(Dated: January 21, 2021) 


\section{Detailed derivation of range-separated correlation}

The short-range correlation corresponds to the effect of electrons when they approach each other, especially at the collision limit. At this limit, the singular Coulomb interaction would lead to a cusp in the wave-function, ${ }^{1-3}$ which closely relates to the dynamic correlation. Thus, a wave-function that fulfills the cusp condition ${ }^{1}$ is necessary for better treatment of the short-range correlation. Here, the correlated wave-function proposed by Colle and Salvetti $(\mathrm{CS})^{4}$ is applied, which takes the following form

$$
\Psi\left(x_{1}, x_{2}, \ldots, x_{N}\right)=\Phi\left(x_{1}, x_{2}, \ldots, x_{N}\right) \prod_{i>j}\left[1-\Lambda\left(\mathbf{r}_{i}, \mathbf{r}_{j}\right)\right]
$$

with

$$
\Lambda\left(\mathbf{r}_{i}, \mathbf{r}_{j}\right)=e^{-\tau^{2} u^{2}}\left[1-\lambda(\mathbf{r})\left(1+\frac{u}{2}\right)\right]
$$

where $x_{i}$ combines both spatial coordinate $\left(\mathbf{r}_{i}\right)$ and spin coordinate $\left(\sigma_{i}\right)$ of the $i$-th electron, $\mathbf{u}=\mathbf{r}_{i}-\mathbf{r}_{j}$, and $\mathbf{r}=\left(\mathbf{r}_{i}+\mathbf{r}_{j}\right) / 2$. $\Phi$ is the one-determinant $\mathrm{HF}$ function, and $\tau$ in Eq $\mathrm{S} 2$ defines the volume of the correlated region around each electron, which is $\tau=q \rho^{1 / 3}(\mathbf{r})$ with the parameter $q$. The determination of $\lambda(\mathbf{r})$ can be found below. With the definition of Eq S1, $\Psi$ would reduce to $\Phi$ when all the electrons are far apart, and it fulfills the cusp condition $^{1}$ when two electrons approach each other. Therefore, this correlated wave-function is a good starting point to derive the short-range correlation functional.

The two-electron density matrix (2-DM) is defined by

$$
\begin{aligned}
& \rho_{2}\left(\mathbf{r}_{1}, \mathbf{r}_{2} ; \mathbf{r}_{1}^{\prime}, \mathbf{r}_{2}^{\prime}\right)=\frac{1}{2} N(N-1) \times \\
& \int \Psi\left(\mathbf{r}_{1} \sigma_{1}, \mathbf{r}_{2} \sigma_{2}, \ldots, x_{N}\right) \Psi^{*}\left(\mathbf{r}_{1}^{\prime} \sigma_{1}, \mathbf{r}_{2}^{\prime} \sigma_{2}, \ldots, x_{N}\right) \prod_{i \geq 3} d \mathbf{r}_{i} \prod_{i \geq 1} d \sigma_{i} .
\end{aligned}
$$

By inserting Eq S1 into Eq S3, and making use of the mean value theorem, 2-DM can be 
effectively approximated by

$$
\begin{aligned}
\rho_{2}\left(\mathbf{r}_{1}, \mathbf{r}_{2} ; \mathbf{r}_{1}^{\prime}, \mathbf{r}_{2}^{\prime}\right)= & \rho_{2}^{\Phi}\left(\mathbf{r}_{1}, \mathbf{r}_{2} ; \mathbf{r}_{1}^{\prime}, \mathbf{r}_{2}^{\prime}\right) \times \\
& {\left[1-\Lambda\left(\mathbf{r}_{1}, \mathbf{r}_{2}\right)-\Lambda\left(\mathbf{r}_{1}^{\prime}, \mathbf{r}_{2}^{\prime}\right)+\Lambda\left(\mathbf{r}_{1}, \mathbf{r}_{2}\right) \Lambda\left(\mathbf{r}_{1}^{\prime}, \mathbf{r}_{2}^{\prime}\right)\right] }
\end{aligned}
$$

where $\rho_{2}^{\Phi}\left(\mathbf{r}_{1}, \mathbf{r}_{2} ; \mathbf{r}_{1}^{\prime}, \mathbf{r}_{2}^{\prime}\right)$ is the HF 2-DM, it can be obtained by inserting the one-determinant HF wave-function into Eq S3.

With Eq S4, a simple form for $\lambda(\mathbf{r})$ can be further derived from the relation between the one-electron density matrix (1-DM) and 2DM, i.e.

$$
(N-1) \rho\left(\mathbf{r}_{1}, \mathbf{r}_{1}^{\prime}\right)=2 \int \rho_{2}\left(\mathbf{r}_{1}, \mathbf{r}_{2} ; \mathbf{r}_{1}^{\prime}, \mathbf{r}_{2}\right) d \mathbf{r}_{2}
$$

Here, 2-DM comes from Eq S4, and 1-DM is approximated by the HF 1-DM, i.e.

$$
\rho\left(\mathbf{r}_{1}, \mathbf{r}_{1}^{\prime}\right) \simeq \rho^{\Phi}\left(\mathbf{r}_{1}, \mathbf{r}_{1}^{\prime}\right)
$$

This assumption is actually effective to derive an appropriate form for $\lambda(\mathbf{r})$. By inserting Eqs S4 and S6 into Eq S5, and setting $\mathbf{r}_{1}^{\prime}=\mathbf{r}_{1}$, we obtain

$$
\int \rho_{2}^{\Phi}\left(\mathbf{r}_{1}, \mathbf{r}_{2}\right)\left[\Lambda^{2}\left(\mathbf{r}_{1}, \mathbf{r}_{2}\right)-2 \Lambda\left(\mathbf{r}_{1}, \mathbf{r}_{2}\right)\right] d \mathbf{r}_{2}=0
$$

where $\rho_{2}^{\Phi}\left(\mathbf{r}_{1}, \mathbf{r}_{2}\right)$ is the diagonal element of $\rho_{2}^{\Phi}\left(\mathbf{r}_{1}, \mathbf{r}_{2} ; \mathbf{r}_{1}^{\prime}, \mathbf{r}_{2}^{\prime}\right)$. Next, making use of Eq S2 and changing the integral variable from $\mathbf{r}_{2}$ to $\mathbf{u}, \mathrm{Eq}$ S7 can be further expanded to

$$
\begin{aligned}
& \int \rho_{2}^{\Phi}\left(\mathbf{r}_{1}, \mathbf{r}_{2}\right) \times \\
& \quad\left\{e^{-2 \tau^{2} u^{2}}\left[1-\lambda(\mathbf{r})\left(1+\frac{u}{2}\right)\right]^{2}-2 e^{-\tau^{2} u^{2}}\left[1-\lambda(\mathbf{r})\left(1+\frac{u}{2}\right)\right]\right\} d \mathbf{u}=0 .
\end{aligned}
$$

As $e^{-2 \tau^{2} u^{2}}$ and $e^{-\tau^{2} u^{2}}$ in the integrand decrease rapidly as $u \rightarrow \infty$, only $\rho_{2}^{\Phi}\left(\mathbf{r}_{1}, \mathbf{r}_{2}\right)$ around 
the points of $\mathbf{r}_{2}=\mathbf{r}_{1}=\mathbf{r}$, i.e. $\rho_{2}^{\Phi}(\mathbf{r}, \mathbf{r})$, need to be taken into account for the integrals in Eq S8. Hence, $\rho_{2}^{\Phi}\left(\mathbf{r}_{1}, \mathbf{r}_{2}\right)$ can be moved out from the integrals without sacrificing much accuracy, which leads to

$$
\begin{aligned}
& \lambda^{2}(\mathbf{r}) \int e^{-2 \tau^{2} u^{2}}\left(1+\frac{u}{2}\right)^{2} d \mathbf{u}+2 \lambda(\mathbf{r}) \int e^{-\tau^{2} u^{2}}\left(1-e^{-\tau^{2} u^{2}}\right)\left(1+\frac{u}{2}\right) d \mathbf{u} \\
& -\int e^{-\tau^{2} u^{2}}\left(2-e^{-\tau^{2} u^{2}}\right) d \mathbf{u}=0
\end{aligned}
$$

The integrals in the above equation can be analytically solved, resulting in the following equation about $\lambda(\mathbf{r})$,

$$
\begin{aligned}
& \lambda^{2}(\mathbf{r})\left(\frac{3}{128 \tau^{2}} \sqrt{\frac{\pi}{2}}+\frac{1}{8 \tau}+\frac{1}{8} \sqrt{\frac{\pi}{2}}\right)+2 \lambda(\mathbf{r})\left(\frac{3}{16 \tau}-\frac{1}{8} \sqrt{\frac{\pi}{2}}+\frac{\sqrt{\pi}}{4}\right) \\
& +\left(\frac{1}{8} \sqrt{\frac{\pi}{2}}-\frac{\sqrt{\pi}}{2}\right)=0 .
\end{aligned}
$$

It is easily to deduce from Eq S10 that

$$
\lim _{\tau \rightarrow 0} \lambda(\mathbf{r}) \simeq \sqrt{\pi} \tau ; \lim _{\tau \rightarrow \infty} \lambda(\mathbf{r})=1-\frac{1}{\sqrt{\pi} \tau}
$$

which thus leads to the simple form for $\lambda(\mathbf{r})$,

$$
\lambda(\mathbf{r}) \simeq=\frac{\sqrt{\pi} \tau}{1+\sqrt{\pi} \tau}
$$

Hence, $\lambda(\mathbf{r})$ depends on the electron density through $\tau$. Eqs S4, S2 and S12 complete the 2-DM needed for the derivation of short-range correlation functional.

The short-range correlation energy can be calculated via

$$
E_{\mathrm{C}}^{\mathrm{sr}}=\int \frac{\operatorname{erfc}(\omega u)}{u}\left[\rho_{2}\left(\mathbf{r}_{1}, \mathbf{r}_{2}\right)-\rho_{2}^{\Phi}\left(\mathbf{r}_{1}, \mathbf{r}_{2}\right)\right] d \mathbf{r}_{1} d \mathbf{r}_{2}
$$

where $\omega$ is the range-separation parameter. By inserting Eq S4 into the above equation, the 
short-range correlation can be further formulated as

$$
\begin{aligned}
E_{\mathrm{C}}^{\mathrm{sr}} & =-\int \rho_{2}^{\Phi}(\mathbf{r}, \mathbf{r}) d \mathbf{r} \int \frac{\rho_{2}^{\Phi}(\mathbf{r}+\mathbf{u} / 2, \mathbf{r}-\mathbf{u} / 2)}{\rho_{2}^{\Phi}(\mathbf{r}, \mathbf{r})} \frac{\operatorname{erfc}(\omega u)}{u} \times \\
& \left\{2 e^{-\tau^{2} u^{2}}\left[1-\lambda(\mathbf{r})\left(1+\frac{u}{2}\right)\right]-e^{-2 \tau^{2} u^{2}}\left[1-\lambda(\mathbf{r})\left(1+\frac{u}{2}\right)\right]^{2}\right\} d \mathbf{u} \\
& =-\int \rho_{2}^{\Phi}(\mathbf{r}, \mathbf{r})\left[\frac{4 \pi}{\tau^{3}} S(\mathbf{r})\right] d \mathbf{r}
\end{aligned}
$$

where

$$
\begin{aligned}
S(\mathbf{r}) & =\frac{\tau^{3}}{4 \pi} \int \frac{\rho_{2}^{\Phi}(\mathbf{r}-\mathbf{u} / 2, \mathbf{r}+\mathbf{u} / 2)}{\rho_{2}^{\Phi}(\mathbf{r}, \mathbf{r})} \frac{\operatorname{erfc}(\omega u)}{u} \times \\
& \left\{2 e^{-\tau^{2} u^{2}}\left[1-\lambda(\mathbf{r})\left(1+\frac{u}{2}\right)\right]-e^{-2 \tau^{2} u^{2}}\left[1-\lambda(\mathbf{r})\left(1+\frac{u}{2}\right)\right]^{2}\right\} d \mathbf{u} .
\end{aligned}
$$

The use of the following relationship

$$
\begin{array}{r}
\int e^{-\tau^{2} u^{2}} A(u, \mathbf{r}) F(\mathbf{u}, \mathbf{r}) d \mathbf{u}=4 \pi F(0, \mathbf{r}) \int_{0}^{\infty} e^{-\tau^{2} u^{2}} A(u, \mathbf{r}) u^{2} d u \\
+\left.\frac{2 \pi}{3} \nabla_{\mathbf{u}}^{2} F(\mathbf{u}, \mathbf{r})\right|_{u=0} \int_{0}^{\infty} e^{-\tau^{2} u^{2}} A(u, \mathbf{r}) u^{4} d u
\end{array}
$$

further leads $S(\mathbf{r})$ to

$$
\begin{aligned}
S(\mathbf{r}) & =\tau^{3} \int \operatorname{erfc}(\omega u) u\left\{2 e^{-\tau^{2} u^{2}}\left[1-\lambda(\mathbf{r})\left(1+\frac{u}{2}\right)\right]\right. \\
& \left.-e^{-2 \tau^{2} u^{2}}\left[1-\lambda(\mathbf{r})\left(1+\frac{u}{2}\right)\right]^{2}\right\} d u \\
& +\frac{\tau^{3}}{6} K \int \operatorname{erfc}(\omega u) u^{3}\left\{2 e^{-\tau^{2} u^{2}}\left[1-\lambda(\mathbf{r})\left(1+\frac{u}{2}\right)\right]\right. \\
& \left.-e^{-2 \tau^{2} u^{2}}\left[1-\lambda(\mathbf{r})\left(1+\frac{u}{2}\right)\right]^{2}\right\} d u \\
& =\frac{A_{1}(z)+A_{2}(z) / \tau+A_{3}(z) W+A_{4}(z) W / \tau}{1+B_{1} / \tau+B_{2} / \tau^{2}}
\end{aligned}
$$

where

$$
K=\frac{\left.\nabla_{\mathbf{u}}^{2} \rho_{2}^{\Phi}(\mathbf{r}-\mathbf{u} / 2, \mathbf{r}+\mathbf{u} / 2)\right|_{u=0}}{\rho_{2}^{\Phi}(\mathbf{r}, \mathbf{r})}
$$


and

$$
W=K / \tau^{2}=4 \frac{\left.\nabla_{\mathbf{u}}^{2} \rho_{2}^{\Phi}(\mathbf{r}-\mathbf{u} / 2, \mathbf{r}+\mathbf{u} / 2)\right|_{u=0}}{\rho^{2}(\mathbf{r}) \tau^{2}} .
$$

Here the relation $\rho_{2}^{\Phi}(\mathbf{r}, \mathbf{r})=\rho^{2}(\mathbf{r}) / 4$ is applied in $K$ of Eq S18. $B_{1}$ and $B_{2}$ are two constants, equal to 1.1284 and 0.3183 respectively. $A_{1}(z), A_{2}(z), A_{3}(z)$, and $A_{4}(z)$ in Eq S17 are functions of $z=\omega / \tau$, which can be effectively approximated by functions of the form of $C \operatorname{erfc}(D z)$. They are 0.1211erfc(0.35z), 0.0459erfc(0.35z), -0.0167erfc(2.0z), and $-0.0102 \operatorname{erfc}(2.0 z)$ respectively. Note that the value of $D$ is 0.35 for both $A_{1}(z)$ and $A_{2}(z)$, and 2.0 for both $A_{3}(z)$ and $A_{4}(z)$. With these, it reasonable to define a more compact form for $S(\mathbf{r})$

$$
S(\mathbf{r})=\frac{c_{1} \operatorname{erfc}\left(D_{1} z\right)+c_{2} \operatorname{erfc}\left(D_{2} z\right) W e^{c_{3} / \tau}}{1+c_{4} / \tau}
$$

with $D_{1}=0.35$ and $D_{2}=2.0$. The insertion of Eq S20 into Eq S14 leads to

$$
\begin{aligned}
E_{\mathrm{C}}^{\mathrm{sr}} & =-\int \rho_{2}^{\Phi}(\mathbf{r}, \mathbf{r})\left[\frac{4 \pi}{\tau^{3}} S(\mathbf{r})\right] d \mathbf{r} \\
& =-4 a \int \frac{\rho_{2}^{\Phi}(\mathbf{r}, \mathbf{r})}{\rho(\mathbf{r})\left(1+d \rho^{-1 / 3}(\mathbf{r})\right)}\left\{\operatorname{erfc}\left(m_{1} \omega \rho^{-1 / 3}(\mathbf{r})\right)+\right. \\
& \left.b \operatorname{erfc}\left(m_{2} \omega \rho^{-1 / 3}(\mathbf{r})\right) \rho^{-8 / 3}(\mathbf{r})\left[\nabla_{\mathbf{u}}^{2} \rho_{2}^{\Phi}\left(\mathbf{r}+\frac{\mathbf{u}}{2}, \mathbf{r}-\frac{\mathbf{u}}{2}\right)\right]_{u=0} e^{-c \rho^{-1 / 3}(\mathbf{r})}\right\} d \mathbf{r}
\end{aligned}
$$

where $m_{1}=D_{1} / q=0.1528, m_{2}=D_{2} / q=0.8734$. And $a=0.04918, b=0.132, c=0.2533$, $d=0.349$. Eq S21 is the short-range part of CS functional.

To further reshape the above functional into a semi-local functional of the electron density, $\rho_{2}^{\Phi}(\mathbf{r}, \mathbf{r})$ and $\left.\nabla_{\mathbf{u}}^{2} \rho_{2}^{\Phi}\left(\mathbf{r}+\frac{\mathbf{u}}{2}, \mathbf{r}-\frac{\mathbf{u}}{2}\right)\right|_{u=0}$ are further processed as below.

First, $\rho_{2}^{\Phi}\left(\mathbf{r}+\frac{\mathbf{u}}{2}, \mathbf{r}-\frac{\mathbf{u}}{2}\right)$ can be formulated in the following form

$$
\begin{aligned}
\rho_{2}^{\Phi}\left(\mathbf{r}+\frac{\mathbf{u}}{2}, \mathbf{r}-\frac{\mathbf{u}}{2}\right) & =\frac{1}{2} \rho\left(\mathbf{r}+\frac{\mathbf{u}}{2}\right) \rho\left(\mathbf{r}-\frac{\mathbf{u}}{2}\right) \\
& -\frac{1}{2}\left|\rho_{1, \alpha}^{\Phi}\left(\mathbf{r}+\frac{\mathbf{u}}{2}, \mathbf{r}-\frac{\mathbf{u}}{2}\right)\right|^{2}-\frac{1}{2}\left|\rho_{1, \beta}^{\Phi}\left(\mathbf{r}+\frac{\mathbf{u}}{2}, \mathbf{r}-\frac{\mathbf{u}}{2}\right)\right|^{2}
\end{aligned}
$$


With this, it is easy to derive that

$$
\rho_{2}^{\Phi}(\mathbf{r}, \mathbf{r})=\frac{1}{2} \rho^{2}(\mathbf{r})-\frac{1}{2} \rho_{\alpha}^{2}(\mathbf{r})-\frac{1}{2} \rho_{\beta}^{2}(\mathbf{r})=\rho_{\alpha}(\mathbf{r}) \rho_{\beta}(\mathbf{r})
$$

and

$$
\left.\nabla_{\mathbf{u}}^{2} \rho_{2}^{\Phi}\left(\mathbf{r}+\frac{\mathbf{u}}{2}, \mathbf{r}-\frac{\mathbf{u}}{2}\right)\right|_{u=0}=2\left[\rho_{\alpha}(\mathbf{r}) t_{\alpha}^{\Phi}(\mathbf{r})+\rho_{\beta}(\mathbf{r}) t_{\beta}^{\Phi}(\mathbf{r})-\rho(\mathbf{r}) t_{W}[\rho(\mathbf{r})]\right]
$$

Here $t_{W}[\rho(\mathbf{r})]$ is a local "Weizsacker" kinetic-energy density

$$
t_{W}[\rho(\mathbf{r})]=\frac{1}{8} \frac{|\nabla \rho(\mathbf{r})|^{2}}{\rho(\mathbf{r})}-\frac{1}{8} \nabla^{2} \rho(\mathbf{r})
$$

and

$$
t_{\alpha}^{\Phi}(\mathbf{r})=\frac{1}{2} t^{\Phi}\left[2 \rho_{\alpha}(\mathbf{r})\right] ; t_{\beta}^{\Phi}(\mathbf{r})=\frac{1}{2} t^{\Phi}\left[2 \rho_{\beta}(\mathbf{r})\right]
$$

with $t^{\Phi}[\rho(\mathbf{r})]$ being the local Hartree-Fock kinetic-energy density

$$
t^{\Phi}[\rho(\mathbf{r})]=\frac{1}{2}\left[\nabla_{\mathbf{r}_{1}} \nabla_{\mathbf{r}_{2}} \rho_{1}^{\Phi}\left(\mathbf{r}_{1}, \mathbf{r}_{2}\right)\right]_{\mathbf{r}_{1}=\mathbf{r}_{2}=\mathbf{r}}-\frac{1}{8} \nabla^{2} \rho(\mathbf{r}) .
$$

By expanding $t^{\Phi}[\rho(\mathbf{r})]$ to second order, i.e.

$$
t^{\Phi}[\rho(\mathbf{r})]=t_{\mathrm{TF}}[\rho(\mathbf{r})]+\frac{1}{9} t_{\mathrm{W}}[\rho(\mathbf{r})]+\frac{1}{18} \nabla^{2} \rho(\mathbf{r})
$$

$\left.\nabla_{\mathbf{u}}^{2} \rho_{2}^{\Phi}\left(\mathbf{r}+\frac{\mathbf{u}}{2}, \mathbf{r}-\frac{\mathbf{u}}{2}\right)\right|_{u=0}$ can be fully expressed as a function of the electron density. $t_{\mathrm{TF}}[\rho(\mathbf{r})]$ here is the Thomas-Fermi kinetic-energy density, $t_{\mathrm{TF}}[\rho(\mathbf{r})]=C_{\mathrm{TF}} \rho^{5 / 3}(\mathbf{r})$ with $C_{\mathrm{TF}}=\frac{3}{10}\left(3 \pi^{2}\right)^{2 / 3}$. 
Finally, the short-range correlation functional takes the following form,

$$
\begin{aligned}
E_{\mathrm{C}}^{\mathrm{sr}}= & -4 a \int \frac{\rho_{\alpha}(\mathbf{r}) \rho_{\beta}(\mathbf{r})}{\rho(\mathbf{r})\left(1+d \rho^{-1 / 3}(\mathbf{r})\right)}\left\{\operatorname{erfc}\left(m_{1} \omega \rho^{-1 / 3}(\mathbf{r})\right)+\right. \\
& 2 b \operatorname{erfc}\left(m_{2} \omega \rho^{-1 / 3}(\mathbf{r})\right) \rho^{-8 / 3}(\mathbf{r})\left[2^{2 / 3} C_{\mathrm{F}} \rho_{\alpha}^{8 / 3}(\mathbf{r})+2^{2 / 3} C_{\mathrm{F}} \rho_{\beta}^{8 / 3}(\mathbf{r})\right. \\
& -\rho(\mathbf{r}) T_{\mathrm{W}}[\rho(\mathbf{r})]+\frac{1}{9}\left(\rho_{\alpha}(\mathbf{r}) T_{\mathrm{W}}\left[\rho_{\alpha}(\mathbf{r})\right]+\rho_{\beta}(\mathbf{r}) T_{\mathrm{W}}\left[\rho_{\beta}(\mathbf{r})\right]\right) \\
& \left.\left.+\frac{1}{18}\left(\rho_{\alpha}(\mathbf{r}) \nabla^{2} \rho_{\alpha}(\mathbf{r})+\rho_{\beta}(\mathbf{r}) \nabla^{2} \rho_{\beta}(\mathbf{r})\right)\right] e^{-c \rho^{-1 / 3}(\mathbf{r})}\right\} d \mathbf{r}
\end{aligned}
$$

Next, $\nabla^{2} \rho(\mathbf{r})$ can be eliminated from Eq S29 by partial integration, so that the final short-range correlation functional can be formulated in the form of GGA, which is

$$
\begin{aligned}
E_{\mathrm{C}}^{\mathrm{sr}}= & -4 a \int \frac{1}{1+d \rho^{-1 / 3}(\mathbf{r})} \frac{\rho_{\alpha}(\mathbf{r}) \rho_{\beta}(\mathbf{r})}{\rho(\mathbf{r})} \operatorname{erfc}\left(m_{1} \omega \rho^{-1 / 3}(\mathbf{r})\right) d \mathbf{r} \\
& -a b \int w(\mathbf{r}) \operatorname{erfc}\left(m_{2} \omega \rho^{-1 / 3}(\mathbf{r})\right)\left\{\rho _ { \alpha } ( \mathbf { r } ) \rho _ { \beta } ( \mathbf { r } ) \left[2^{11 / 3} C_{\mathrm{F}}\left(\rho_{\alpha}^{8 / 3}(\mathbf{r})+\rho_{\beta}^{8 / 3}(\mathbf{r})\right)\right.\right. \\
& +\left(\frac{47}{18}-\frac{7}{18} \delta(\mathbf{r})\right)|\nabla \rho(\mathbf{r})|^{2}-\left(\frac{5}{2}-\frac{1}{18} \delta(\mathbf{r})\right)\left(\left|\nabla \rho_{\alpha}(\mathbf{r})\right|^{2}+\left|\nabla \rho_{\beta}(\mathbf{r})\right|^{2}\right) \\
& \left.-\frac{\delta(\mathbf{r})-11}{9}\left(\frac{\rho_{\alpha}(\mathbf{r})}{\rho(\mathbf{r})}\left|\nabla \rho_{\alpha}(\mathbf{r})\right|^{2}+\frac{\rho_{\beta}(\mathbf{r})}{\rho(\mathbf{r})}\left|\nabla \rho_{\beta}(\mathbf{r})\right|^{2}\right)\right]-\frac{2}{3} \rho^{2}(\mathbf{r})|\nabla \rho(\mathbf{r})|^{2} \\
& \left.+\left(\frac{2}{3} \rho^{2}(\mathbf{r})-\rho_{\alpha}^{2}(\mathbf{r})\right)\left|\nabla \rho_{\beta}(\mathbf{r})\right|^{2}+\left(\frac{2}{3} \rho^{2}(\mathbf{r})-\rho_{\beta}^{2}(\mathbf{r})\right)\left|\nabla \rho_{\alpha}(\mathbf{r})\right|^{2}\right\} d \mathbf{r} \\
& -a b \int w(\mathbf{r}) \eta(\mathbf{r}) \rho_{\alpha}(\mathbf{r}) \rho_{\beta}(\mathbf{r})\left[\frac{7}{3} \rho(\mathbf{r}) \nabla \rho_{\alpha}(\mathbf{r}) \nabla \rho_{\beta}(\mathbf{r})\right. \\
& \left.+\left(\rho(\mathbf{r})+\frac{1}{3} \rho_{\alpha}(\mathbf{r})\right)\left|\nabla \rho_{\alpha}(\mathbf{r})\right|^{2}+\left(\rho(\mathbf{r})+\frac{1}{3} \rho_{\beta}(\mathbf{r})\right)\left|\nabla \rho_{\beta}(\mathbf{r})\right|^{2}\right] d \mathbf{r}
\end{aligned}
$$

where $w(\mathbf{r})=\frac{e^{-c \rho^{-1 / 3}(\mathbf{r})}}{1+d \rho^{-1 / 3}(\mathbf{r})} \rho^{-11 / 3}(\mathbf{r}), \delta(\mathbf{r})=c \rho^{-1 / 3}(\mathbf{r})+\frac{d \rho^{-1 / 3}(\mathbf{r})}{1+d \rho^{-1 / 3}(\mathbf{r})}$, and $\eta(\mathbf{r})=-\frac{2}{3 \sqrt{\pi}} m_{2} \omega e^{-m_{2}^{2} \omega^{2} \rho^{-2 / 3}(\mathbf{r})} \rho^{-4 / 3}(\mathbf{r})$. The long-range correlation functionals can be derived similarly, whose forms can be obtained by replacing complementary error functions in both Eqs S21 and S30 with error functions and multiplying the last integral in Eq S30 by -1 . 


\section{Computational details}

In this work, all calculations were carried out with a local version of NorthWest Computational Chemistry (NWChem) software package. ${ }^{5}$ The test sets used were $62-97^{6}$ for atomization energies, HTBH38/08 and NHTBH38/08 ${ }^{7,8}$ for reaction barriers, G2RC ${ }^{9}$ for reaction energies, and NCCE31 $/ 05^{8}$ for noncovalent complexation energies. B3LYP $/ 6-311 \mathrm{G}(\mathrm{d}, \mathrm{p})$ was used to optimize geometries for the Diels-Alder reaction of 1,3-butadiene and ethylene. The basis set $6-311++\mathrm{G}(3 \mathrm{df}, 3 \mathrm{pd})^{10-12}$ was used for all the energy calculations except that augcc-pV5Z ${ }^{13}$ was used for the calculation of the correlation energy of He atom.

(a) RB

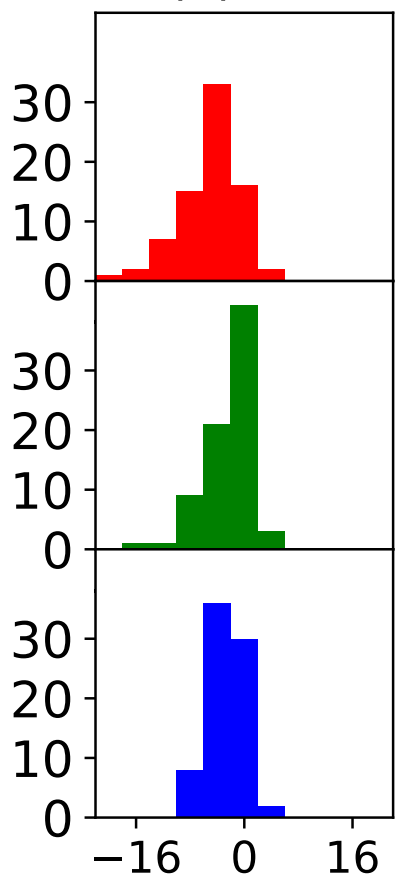

(b) G2RC

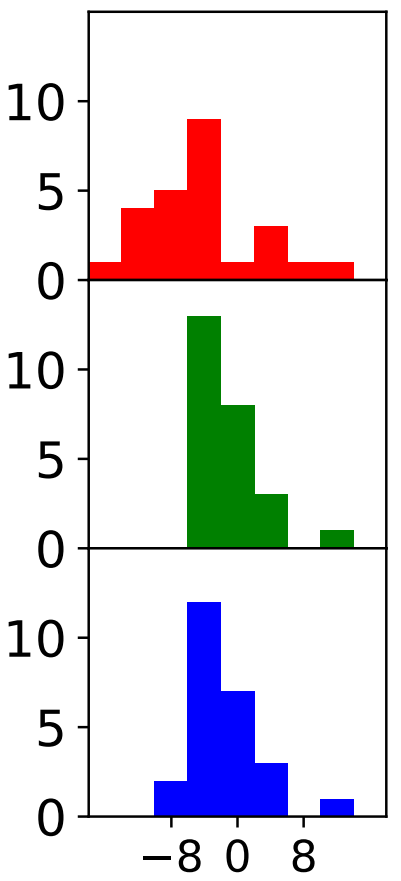

(c) NCCE

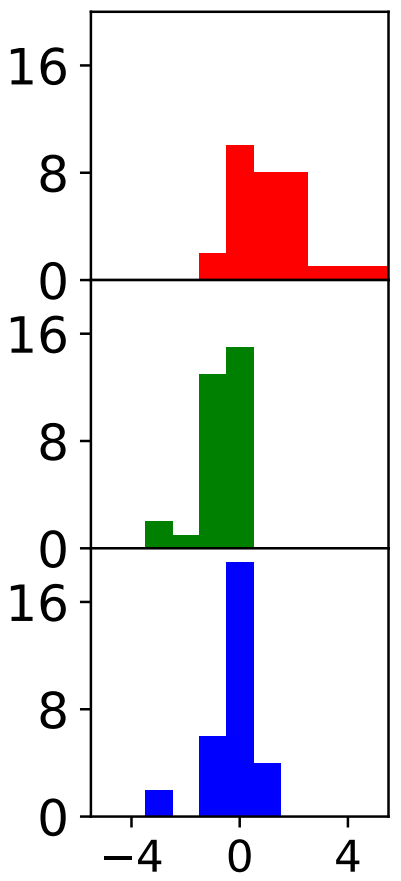

Figure S1: Error distributions for the tests of reaction barriers, reaction energies, and noncovalent complexation energies. LC-BLYP (red), LC-BLYPr (green), and CAM-B3LYP (blue) are checked. The test sets are (a) HTBH38/08 and NHTBH38/08 7,8 for reaction barriers, (b) $\mathrm{G} 2 \mathrm{RC}^{9}$ for reaction energies, and (c) NCCE31 $/ 05^{8}$ for noncovalent complexation energies. The units are $\mathrm{kcal} / \mathrm{mol}$ for all the $\mathrm{x}$ axes. LC-BLYP shows large errors for many systems. Without the long-range correlation energy LYPlr, LC-BLYPr shows much narrow error distributions. 
For a more complete analysis, here we further release the parameter $\omega$ in the $\mathrm{XC}$ functional, and optimize it together with $c_{\mathrm{lr}}$ against the same fitting set (i.e. the G2/97 set). The resulting parameters are $c_{\mathrm{lr}}=0.09$ and $\omega=0.38$, which are slightly different from the values used in the main text (i.e. $c_{\mathrm{lr}}=0.00$ and $\omega=0.33$ ). These new values for $c_{\mathrm{lr}}$ and $\omega$ can further reduce the MAEs of the atomization energies in the fitting set by about $2 \mathrm{kcal} / \mathrm{mol}$, however, the performance for reaction barriers and noncovalent complexation energies becomes worse, the MAEs are 3.60 and $0.96 \mathrm{kcal} / \mathrm{mol}$ respectively, which are larger than 2.95 and $0.73 \mathrm{kcal} / \mathrm{mol}$ by LC-BLYPr in the main text. In addition, these test results further support the conclusion in the present work, the small contribution of LYPlr in the XC functional again indicates that LYPlr cannot effectively capture the long-range correlation effect in LC-BLYP.

\section{References}

(1) Bingel, W. A. A Physical Interpretation of the Cusp Conditions for Molecular Wave Functions. Theor. Chem. Acc. 1967, 8, 54-61.

(2) Chapman, J. A.; Chong, D. P. Cusp Constraints for Atomic Wavefunctions. Can. J. Chem. 1970, 48, 2722-2726.

(3) Kimball, J. C. Short-Range Correlations and Electron-Gas Response Functions. Phys. Rev. A 1973, 7, 1648-1652.

(4) Colle, R.; Salvetti, O. Approximate Calculation of the Correlation Energy for the Closed and Open Shells. Theoret. Chim. Acta 1979, 53, 55-63.

(5) Valiev, M.; Bylaska, E. J.; Govind, N.; Kowalski, K.; Straatsma, T. P.; Dam, H. J. J. V.; Wang, D.; Nieplocha, J.; Apra, E.; Windus, T. L. NWChem: A comprehensive and scalable open-source solution for large scale molecular simulations. Comput. Phys. Commun. 2010, 181, 1477-1489. 
(6) Curtiss, L. A.; Raghavachari, K.; Redfern, P. C.; Pople, J. A. Assessment of Gaussian-2 and Density Functional Theories for the Computation of Enthalpies of Formation. J. Chem. Phys. 1997, 106, 1063-1079.

(7) Zheng, J.; Zhao, Y.; Truhlar, D. G. The DBH24/08 Database and Its Use to Assess Electronic Structure Model Chemistries for Chemical Reaction Barrier Heights. J. Chem. Theory Comput. 2009, 5, 808-821.

(8) Peverati, R.; Truhlar, D. G. Quest for a Universal Density Functional: the Accuracy of Density Functionals Across a Broad Spectrum of Databases in Chemistry and Physics. Philos. Trans. Royal. Soc. A 2014, 372, 20120476.

(9) Goerigk, L.; Hansen, A.; Bauer, C.; Ehrlich, S.; Najibi, A.; Grimme, S. A Look at the Density Functional Theory Zoo with the Advanced GMTKN55 Database for General Main Group Thermochemistry, Kinetics and Noncovalent Interactions. Phys. Chem. Chem. Phys. 2017, 19, 32184-32215.

(10) Krishnan, R.; Binkley, J. S.; Seeger, R.; Pople, J. A. Self-Consistent Molecular Orbital Methods. XX. A Basis Set for Correlated Wave Functions. J. Chem. Phys. 1980, 72, $650-654$.

(11) Frisch, M. J.; Pople, J. A.; Binkley, J. S. Self-Consistent Molecular Orbital Methods 25. Supplementary Functions for Gaussian Basis Sets. J. Chem. Phys. 1984, 80, 3265-3269.

(12) Clark, T.; Chandrasekhar, J.; Spitznagel, G. W.; Schleyer, P. V. R. Efficient Diffuse Function-Augmented Basis Sets for Anion Calculations. III. The 3-21+G Basis Set for First-Row Elements, Li-F. J. Comput. Chem. 1983, 4, 294-301.

(13) Dunning, T. H. Gaussian Basis Sets for Use in Correlated Molecular Calculations. I. The Atoms Boron Through Neon and Hydrogen. J. Chem. Phys. 1989, 90, 1007-1023. 